\title{
Implementation of bending-active elements in kinematic form-active structures - Part I: Design of a representative case study
}

\author{
Silke Puystiens, ${ }^{\mathrm{a}, \mathrm{b}, *}$, Maarten Van Craenenbroeck ${ }^{\mathrm{b}}$, Danny Van Hemelrijck ${ }^{\mathrm{a}}$, Wim Van Paepegem ${ }^{\mathrm{c}}$, \\ Marijke Mollaert ${ }^{\mathrm{b}}$, Lars De Laet ${ }^{\mathrm{b}}$ \\ a Department of Mechanics of Materials and Constructions, Vrije Universiteit Brussel, Pleinlaan 2, 1050 Brussels, Belgium \\ b Department of Architectural Engineering, Vrije Universiteit Brussel, Pleinlaan 2, 1050 Brussels, Belgium \\ c Department of Materials Science and Engineering, Ghent University, Technologiepark-Zwijnaarde 903, 9052 Zwijnaarde, \\ Belgium \\ * Corresponding author: silke.puystiens@vub.ac.be
}

\begin{abstract}
Due to their low self-weight and their inherently high flexibility, technical textiles offer great possibilities for the integration in kinematic structures. Furthermore, the implementation of active bending in a transformable design creates new challenging perspectives.

The paper describes an integrated approach for transformable textile hybrids where an improved design is obtained through a parameter study, performing a structural analysis in the different phases of the deployment. The studied parameters include (i) the form-finding position, (ii) the prestress (ratio), (iii) the used materials and sections (including the fibre directions) and (iv) the number of bending-active elements.

This research confirms the feasibility of realizing kinematic form-active structures with integrated bending-active elements, where both the membrane and the supporting structure are stable in the different configurations. Due to the high interaction between the bending-active supporting system and the pretensioned membrane, the different parameters influence each other significantly.

In a next step, an experimental verification of the designed pringle-shaped textile hybrid is carried out in order to both confirm the possibilities and reveal the remaining challenges.
\end{abstract}

Keywords: Tensile fabric structures; Kinematic structures; Active bending; Structural design; Numerical modelling 


\section{Introduction}

Engineers and architects are persistently striving for a more sustainable built environment, which can respond to the demand for more adaptable building solutions with a high level of structural efficiency. Both covering spaces for weather protection and improving the energy performance of (already existing) buildings ask for dynamic and light architectural solutions.

Lightweight technical textiles offer great possibilities for the integration in transformable structures. However, until present most membrane structures remain static or are only structurally stable (i.e. properly tensioned) in their fully deployed state. The challenge of this research is to verify the feasibility of realizing kinematic form-active structures (KFAS), where both the membrane and the supporting structure are stable in the different geometrical configurations.

Previous research explored the design and structural behaviour of a kinematic membrane structure that is attached to a hinged rigid frame [1]. The research described in this paper, forms the next step in the analysis of kinematic membrane structures, where the interaction between the membrane and the boundary elements complicates the structural behaviour and analysis.

Combining bending-active (BA) elements with textile materials offers great possibilities to create innovative designs and structurally efficient hybrid structures [2][3]. The integrated bending-active elements can serve both as a supporting structure and as a shape-defining structural element. Compared to traditional membrane structures, the number of external supports can thus be reduced. Furthermore, the membrane's tension force results in an important increase in structural stability under loading and reduces the risk of buckling of the beam elements [4]. By adding the prestressed membrane, a more homogeneous curvature of the bending-active element will be obtained, resulting in a more uniform stress distribution. All these advantages allow the use of very slender lightweight profiles and thus prove the structural efficiency of these structures, that are also known as 'textile hybrids' [5].

When adding the kinematic aspect of the abovementioned BA KFAS, another interesting typology arises where the bending (and torsion) of the elements is used as a controlling mechanism to avoid loss of fabric tension during the deployment.

The current study analyses the feasibility of a case study, combining lightweight components (bending active linear elements, cables and membrane), evaluating the influence of the design parameters and verifying the structural behaviour under deployment.

\section{Description of the case study}

\subsection{Structural concept}

For this research, a seemingly simple pringle-shaped structure is investigated, consisting of a bendingactive ring with a membrane tensioned inside the ring, forming a self-stabilizing system. A parameter study is performed to investigate under which conditions this structure can transform and how its structural response can be improved during all the phases of the deployment (within the intended range). The important interaction between the prestressed membrane and the bending-active boundary element, leads to a dependency between the different studied parameters, which consequently complicates the interpretation of the results.

In this case study, the beam is first bent from a linear element towards a circular ring, which initiates bending stresses in the beam element. Tensioning the initially flat membrane into the ring results in a 
three-dimensional pringle-shape: the more the membrane is pretensioned, the higher the overall curvature of the structure becomes. Starting from this pringle-shaped membrane structure, i.e. the formfound geometry, the high points are pulled towards each other to achieve the kinematic deployment (Figure 1).
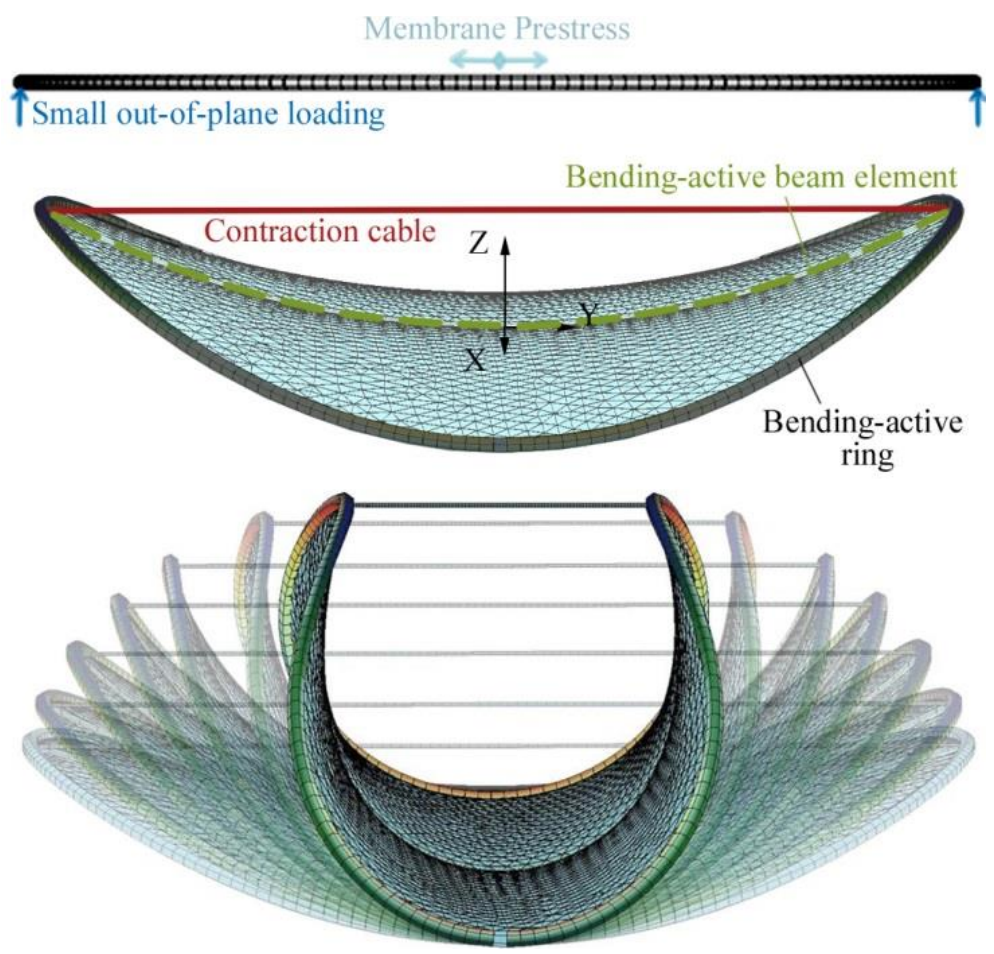

Figure 1. General principles of the structural behaviour of the kinematic textile hybrid; (i) initial flat state; (ii) popped up 3D state under a certain membrane prestress (form-finding) and (iii) kinematic deployment of the structure (simulated in Sofistik (C))

\subsection{Material properties}

\subsubsection{Polyester fabrics}

To show the importance of the selected membrane material (see section 5.1.1), a comparison between a relatively stiff PVC-coated membrane and a highly flexible PU-coated membrane is conducted (both provided by Sioen [6]).

The PU-coated knitted polyester fabric (type F5637) has a mass density of $290 \mathrm{~g} / \mathrm{m}^{2}$ and a thickness of $\pm 0.43 \mathrm{~mm}$. The tensile strength in warp direction is $400 \mathrm{~N} / 5 \mathrm{~cm}$ and in weft direction $300 \mathrm{~N} / 5 \mathrm{~cm}$. The PVC-coated polyester fabric (type T2103) has a thickness of $0.83 \mathrm{~mm}$ and a mass density of $900 \mathrm{~g} / \mathrm{m}^{2}$. The fabric has a breaking strength of $4000 \mathrm{~N} / 5 \mathrm{~cm}$ in both warp and weft direction.

Both uniaxial and biaxial tests are performed at the Vrije Universiteit Brussel. Using the stress and strain data from the biaxial tests, the elastic constants are calculated. The applied material model is the one proposed by Galliot and Luchsinger, where the elastic properties can vary with the applied load ratio, assuming that the material is orthotropic linear elastic within one particular load ratio [7]. The derived material properties are presented in Table 1. 
Table 1. Material properties for the PVC polyester fabric and the PU polyester fabric determined following the model of Galliot and Luchsinger [7]

\begin{tabular}{c|c|c|c|c|c|c} 
& $E_{w}(k N / m)$ & $E_{f}(k N / m)$ & $\Delta_{E w}(k N / m)$ & $\Delta_{E f}(k N / m)$ & $v_{w f}(-)$ & $G_{w f}(k N / m)$ \\
\hline PVC PES & 663 & 557 & 384 & 149 & 0.39 & 22.4 \\
\hline PU PES & 6.65 & 4.56 & 0.00 & 3.72 & 0.49 & 1.52
\end{tabular}

\subsubsection{Glass Fibre Reinforced Polymers (GFRPs)}

The Glass Fibre Reinforced Polymer (GFRP) elements are provided by Vink [8]. The circular bar elements consist of unidirectional fibres only, whereas the rectangular beam elements consist of both unidirectional fibres and multidirectional fibre mats.

The used circular elements (serving as the boundary ring) have a diameter of $12.7 \mathrm{~mm}$ and are subjected to both uniaxial tensile tests and three point bending tests. The tensile modulus equals $46 \mathrm{GPa}$ and the bending modulus $41 \mathrm{GPa}$. The used rectangular elements (serving as internal bending-active elements) have a width of $40 \mathrm{~mm}$ and a thickness of $5 \mathrm{~mm}$. The tensile modulus measured from the uniaxial tests equals $35 \mathrm{GPa}$. The other material properties are adopted from Fiberline Design Guide [9] (see Table 2).

\begin{tabular}{c|c|c|c|c}
\multicolumn{5}{c}{ Table 2. Important material properties for the GFRP rods and beams } \\
& $E_{0}(G P a)$ & $E_{90}(G P a)$ & $v_{0}(-)$ & $v_{90}(-)$ \\
\hline GFRP rod & 41 & 7 & 0.23 & 0.09 \\
\hline GFRP beam & 35 & 7 & 0.23 & 0.09
\end{tabular}

\section{Numerical simulation}

Although current research investigates how to model the complex nonlinear behaviour of membrane materials numerically (e.g. [10]), for this study a simple linear elastic material model is adopted. The design and analysis of the studied textile hybrid occurs through Finite Element Modelling in Sofistik [11], using the Galliot \& Luchsinger material model described above.

In this case study the ring element is shaped depending on the applied prestress in the membrane, which is also called the simultaneous approach [5], performing the form-finding of the membrane and the beam elements at the same time.

Due to the complex interaction between the membrane and bending elements and, consequently, the large deformations of the structure when increasing the membrane's prestress, the form-finding process of this textile hybrid is conducted slightly different compared to conventional membrane structures. In Sofistik, the form-finding of the membrane with a certain applied prestress requires to temporarily reduce the membrane stiffness, which leads to unacceptable distortions of the outer membrane elements when the bending-active boundary element deforms too much.

The following process is adopted to perform the form-finding in Sofistik in order to avoid these unacceptable distortions; the two lower points can still slide towards each other, after which the resulting cutting pattern can be generated (also visualized in Figure 2): 


\section{THE PRELIMINARY FORM-FINDING (1)}

Defining an 'approximate' shape of the structure by applying the membrane's prestress (with full membrane stiffness). Due to the settlement of the ring during this pretensioning, i.e. the outof-plane movement, the resulting stresses in the membrane are often much lower than the intended prestress;

\section{THE ACTUAL FORM-FINDING (2)}

Performing the actual form-finding with a reduced membrane stiffness on the geometry obtained in step 1, keeping the ring element fixed. This results in a homogeneously distributed prestress that equals the intended value;

\section{THE FINAL FORM-FINDING (3)}

Ending the form-finding with the full membrane stiffness to relax the membrane. In this case, releasing the constraints on the ring results in the further settlement of the ring as expected. As the ring undergoes further relaxation, the membrane prestress slightly drops under the intended prestress value.

After the form-finding, the support points are fully fixed and the structure is subjected to an analysis under external loading:

\section{KINEMATIC DEPLOYMENT (4)}

In this case, this external loading represents the kinematic deployment of the textile hybrid. As shown in Figure 2, this kinematic deployment is established through the contraction of an elastic cable between the two upper points of the pringle shape (the elastic cable approach described in [5] will be applied).

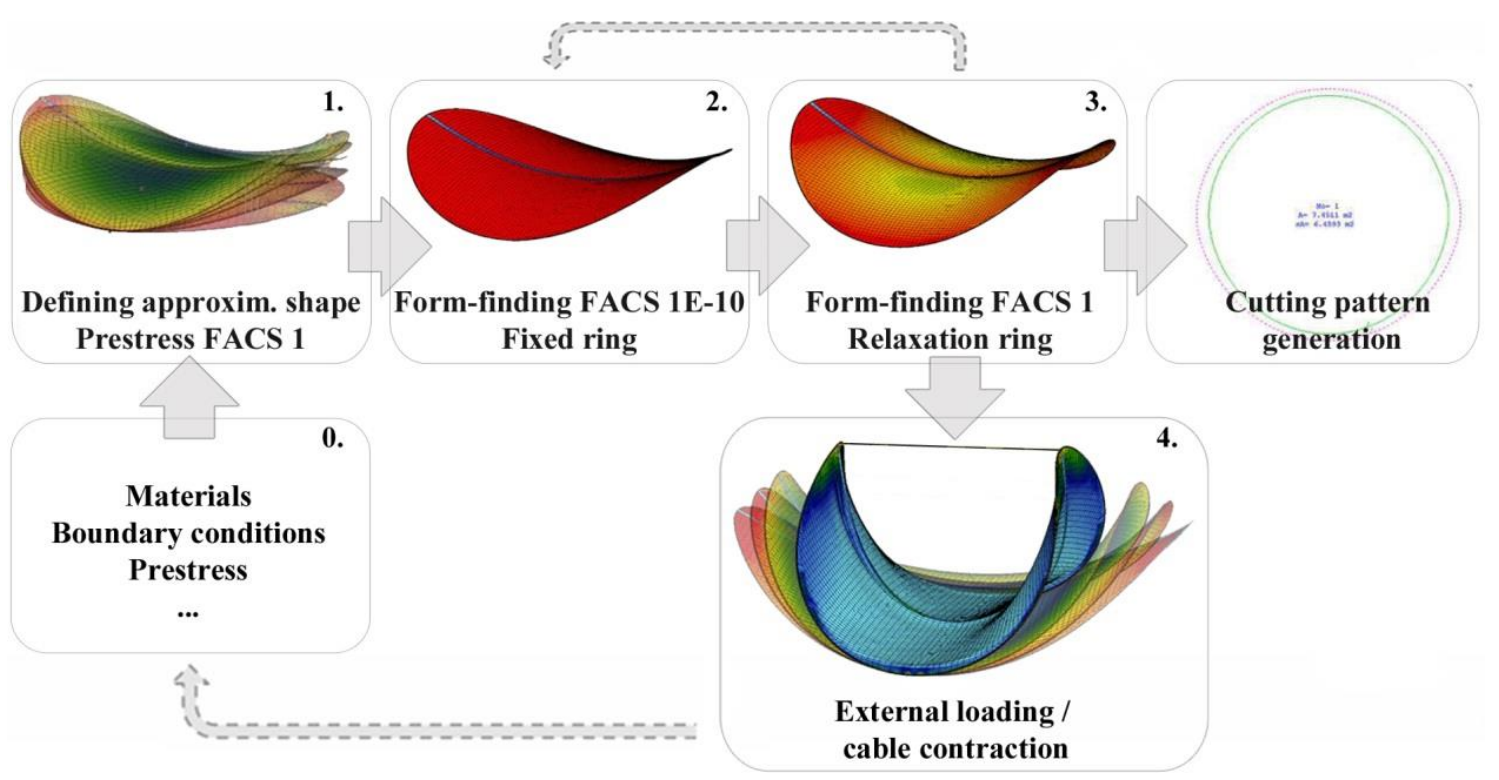

Figure 2. Methodology for the form-finding of the textile hybrid to finally come to the cutting pattern; from this form-found structure, the kinetic deployment is initiated (FACS represents the membrane stiffness factor in Sofistik) 


\section{Parameter study}

\subsection{The need for a parameter study}

The complex interaction between membrane and bending elements complicates the structural analysis of textile hybrids. Changes in e.g. element dimensions and prestress values already influence the geometry in the form-finding stage, but the behaviour during the kinetic deployment varies as well with these initial design choices.

Based on the structural analysis of the numerical model, one can suggest varying design parameters and thus determine the influence of each of the selected parameters. This iterative process allows improving the overall structural behaviour of the textile hybrid, aiming for a structurally stable system in all the phases of its deployment.

In order to quantify the effect of each parameter, the structural stability of the system is verified, measuring forces and stresses in the elements. Furthermore, the system's deflections are measured under a certain applied external loading, also providing an indication of the structure's overall stiffness. Of course, it is also verified that the resulting stresses never exceed the materials' limits.

\section{2. $\quad$ Studied parameters}

The chosen materials and dimensions have a great influence on the form-finding geometry and thus also on the behaviour during the kinetic deployment. GFRP elements are highly suited for use as bendingactive elements due to their high strength and low bending stiffness. In the scope of this research, no other materials are considered for the bending elements; only the influence of varying sections is investigated. For the membrane material, on the contrary, two types of fabric are compared: a relatively stiff PVC-coated polyester fabric and a stretchable PU-coated polyester fabric.

As the used membrane materials consist of two orthogonal fibre directions with different properties, the orientation of the membrane is of remarkable importance. Three variants are investigated: (i) with the warp direction aligned along the line connecting the top points $\left(0^{\circ}\right)$, (ii) with the warp direction aligned along the line connecting the lower points $\left(90^{\circ}\right)$ and (iii) at $45^{\circ}$.

The applied prestress in the membrane contributes to the overall stiffness of the tensile structure. Also, the prestress in the membrane influences the form-finding geometry, as increasing the prestress also results in an increase in curvature (and thus in geometric stiffness for this particular prestress). Choosing the prestress value will depend on the selected profile sections and vice versa. It is thus important to evaluate these choices thoroughly. Furthermore, it is possible to apply an asymmetric prestress ratio depending on the resulting stress distributions in the different phases of the deployment.

Initially, the structure consists only of a GFRP ring element wherein a membrane is tensioned. To improve the structural behaviour of the pringle shape, i.e. reduce the loss of pretension during the kinematic deployment, a new GFRP element is added internally. The differences without and with an internal element are measured, but also the effect of different modelling methods for the connection of the internal element to the membrane. 


\section{Results and discussion}

Note that the selected values and properties take into account that the experimental verification of this model should be performed manually and that forces and stresses are thus limited to what can be applied by hand [13].

The study starts from a ring with a diameter of $3.18 \mathrm{~m}$ - with a circular profile section of $12.7 \mathrm{~mm}$. The GFRP's bending modulus of $41 \mathrm{GPa}$ results in initial bending stresses in the ring element of:

$$
\sigma=\frac{E \cdot t}{2 r}=163.5 \mathrm{MPa}
$$

with $\mathrm{E}$ the bending modulus, $\mathrm{t}$ the section's diameter and $\mathrm{r}$ the ring's radius.

Initially, a PVC-coated polyester fabric is tensioned in the GFRP ring with its warp direction aligned along the y-axis (x-and y-axis like defined in Figure 5). In the form-finding stage, the two lower points can slide along this $\mathrm{x}$-axis, i.e. towards and away from each other, to allow the ring element to transform to its pringle-shaped geometry. Once the kinematic deployment is initiated these points are fully fixed.

\subsection{Materials and sections}

\subsubsection{Membrane material}

Using a PVC-coated polyester fabric requires a higher initial prestress than using flexible PU-coated fabric to obtain a stable textile hybrid. This is due to the high flexibility of the bending-active ring, which causes a reduction in membrane strains as the ring relaxes under the applied prestress. For the flexible membrane, this reduction in membrane strains only results in a slight decrease in membrane stresses, whereas the stiffer PVC fabric exhibits a significant drop in stresses. This can also be seen in the results from the uniaxial tensile tests on both materials in Figure 3.

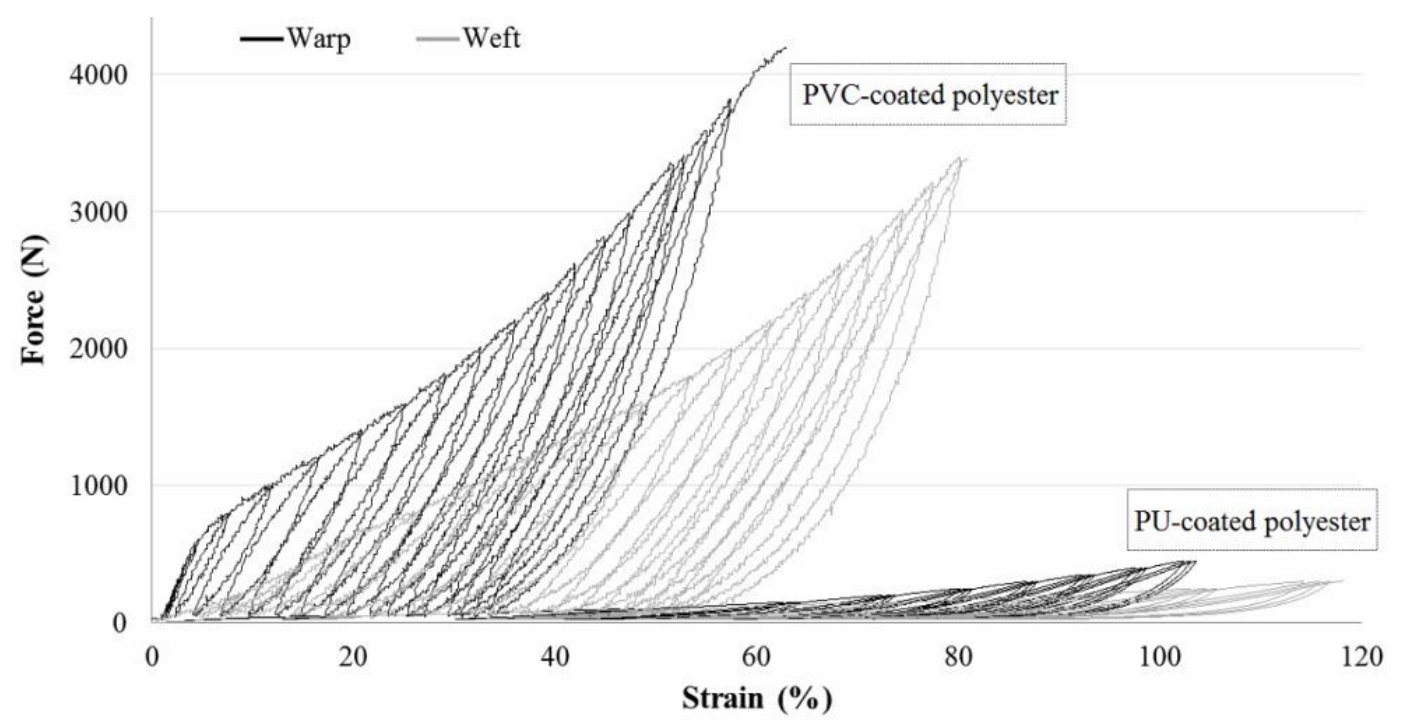

Figure 3. Force-displacement curves from uniaxial tensile tests on both the PVC-coated and the PU-coated polyester fabric 
In order to obtain a similar geometry for the form-finding with both materials, the initial prestress value should be set at $0.3 \mathrm{kN} / \mathrm{m}$ for the PU fabric and at $11 \mathrm{kN} / \mathrm{m}$ for the PVC fabric. Figure 4 shows that (at the final form-finding state) the stresses in the membrane are distributed more uniformly in the PU fabric, as compared to the PVC fabric. The actual prestress in the middle of the membrane after the final form-finding lies at $\pm 0.10 \%$ of the intended prestress $(=0.01 / 11 \mathrm{kN} / \mathrm{m})$ for the PVC PES and at $33 \%$ $(=0.10 / 0.3 \mathrm{kN} / \mathrm{m})$ for the PU PES. Note that the colour scale is different for each case.

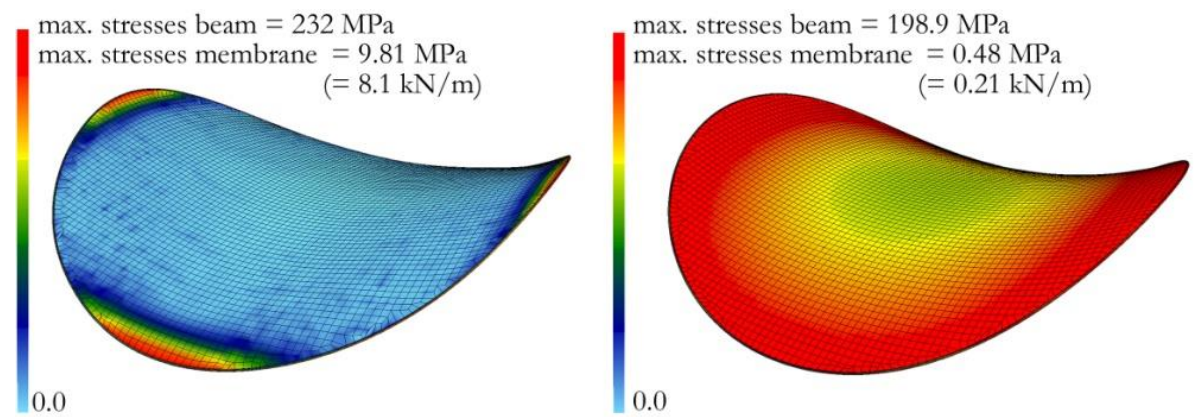

Figure 4. Von Mises stresses in final the form-finding state, after relaxation: (i) for the PVC-coated polyester fabric and (ii) for the PU-coated polyester fabric

The beam stresses presented in Figure 4 incorporate both the initial bending stress and the stresses resulting from the form-finding. The given Von Mises stresses represent the maximum stresses on the ring's section.

Figure 5 zooms in on the results after the final form-finding along two perpendicular lines. Both membrane forces $(\mathrm{kN} / \mathrm{m})$ in $\mathrm{x}$ - and y-direction are plotted for the PVC- and the PU-coated membrane.

For both membrane materials, the forces in the middle are similar in $\mathrm{x}$ - and $\mathrm{y}$-direction and increase when approaching the boundary ring. Along the vertical line, i.e. the y-direction, the forces in $\mathrm{x}$-direction increase more towards the top points than the forces in y-direction. Along the horizontal line on the contrary, the opposite occurs: the forces in y-direction are larger than in x-direction when approaching the lower points.

These force concentrations can again be explained by the corresponding strains in the membrane. As previously mentioned, the large deformations of the ring element cause a decrease in strain and consequently lead to a loss of membrane forces. Close to the boundary ring, i.e. where the chord of the ring is very small (in $\mathrm{x}$ - or $\mathrm{y}$-direction), only limited straining is possible and the drop in membrane forces is thus also limited. Near the lower points the forces in y-direction are thus higher, whereas in the top points the $\mathrm{x}$-direction shows higher forces. Comparing the graphs from the PVC and the PU PES illustrates that the high local stress concentrations are avoided when using the more flexible material. 

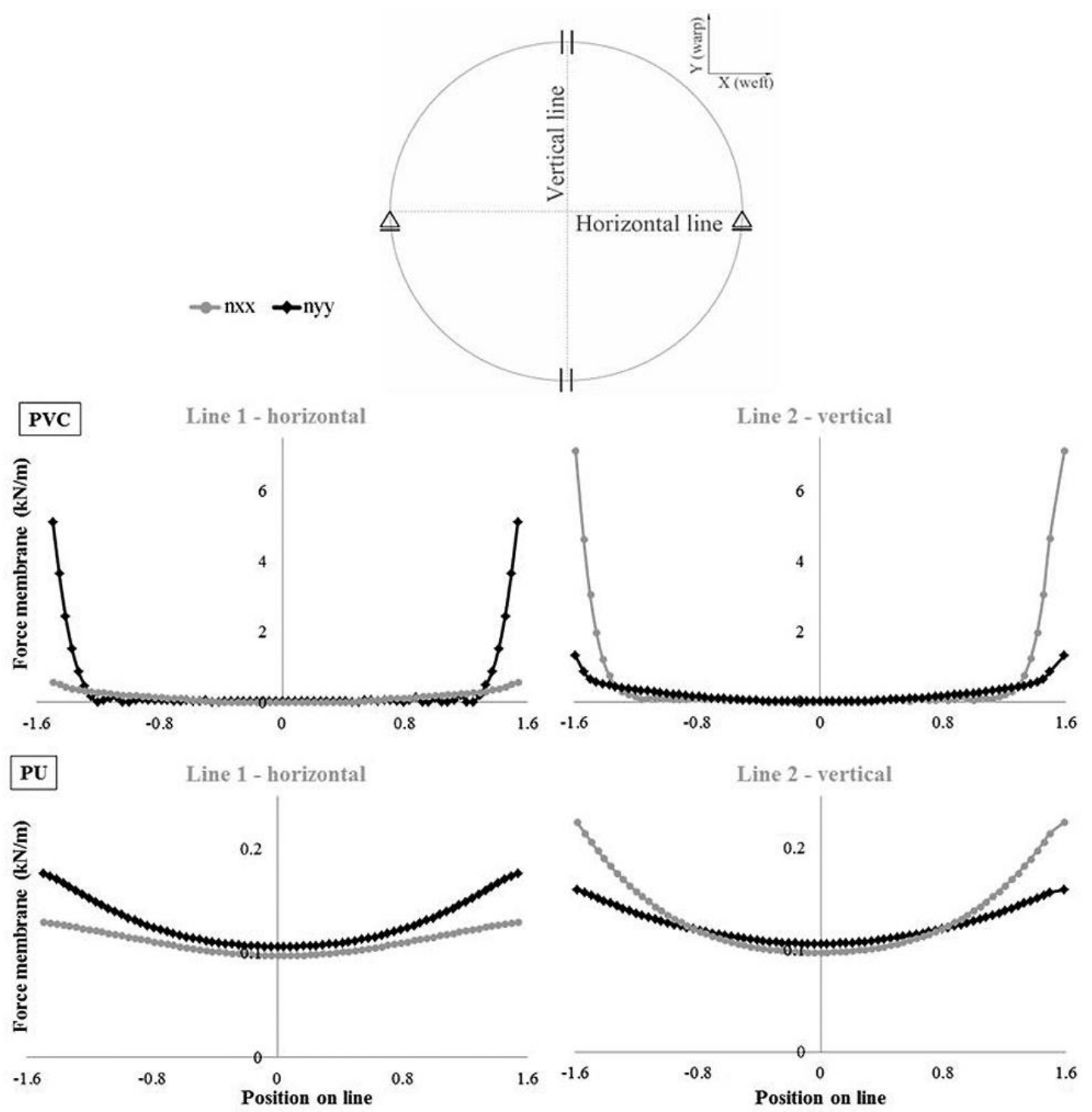

Figure 5. After the final form-finding: Membrane forces $(\mathrm{kN} / \mathrm{m})$ along the axes of symmetry for the PVC-and the PU-coated membrane; nxx is grey, nyy is black

This first part of the analysis compares the structural result of the form-finding, but also the influence on the behaviour during the kinematic deployment is of major importance.

Contracting the PVC PES structure obtained in Figure 4 would not clearly show the influence of shortening the contraction cable on the membrane stresses, as these are already unacceptably low after the form-finding. To obtain a pringle-shaped form-found structure with a PVC membrane prestress of $\pm 0.1 \mathrm{kN} / \mathrm{m}$ in the centre, the stiffness of the bending-active supporting structure should be increased, as explained in section 5.1.2. With a ring section of $16 \mathrm{~mm}$ diameter and a membrane prestress of $6.5 \mathrm{kN} / \mathrm{m}$, a similar pringle geometry is obtained after form-finding. From this state, the contraction cable is gradually shortened and the membrane forces in $\mathrm{x}$ - and y-direction are plotted for both the PVC and the PU PES membranes (see Figure 6). From the graph it is clear that the forces in the membrane drop much quicker for the stiffer PVC coated fabric and thus also here the advantages of using a flexible membrane material are highlighted. 


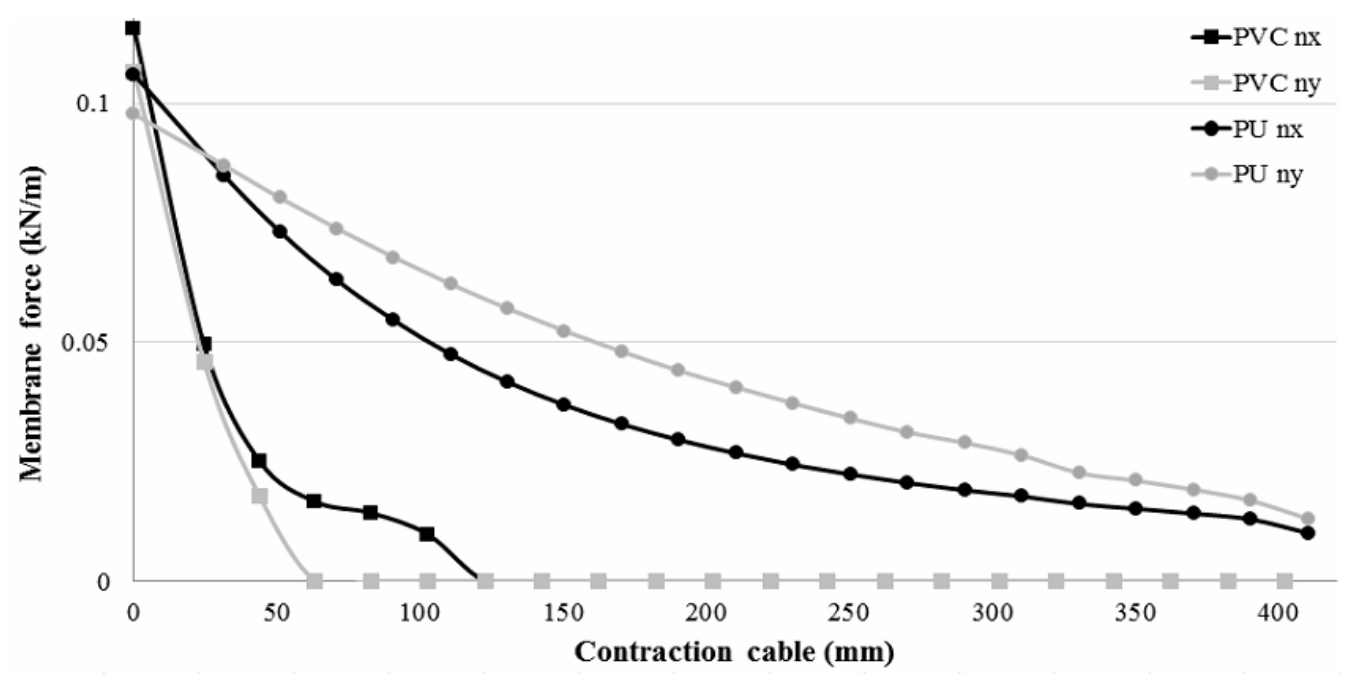

Figure 6. Evolution of the membrane forces (in the centre of the membrane) during the contraction of the cable: comparison between the PU- and the PVC-coated polyester

For further investigation of the pringle shaped textile hybrid only the flexible PU-coated polyester fabric is considered.

\subsubsection{Beam section}

Increasing the ring's section results in an increase in overall stiffness of the system and thus in a reduced out-of-plane movement for a given prestress. Figure 7 plots the height of the top points (z-direction) during the different phases of the form-finding process. The prestress value of $0.3 \mathrm{kN} / \mathrm{m}$ is applied gradually, with steps of $0.03 \mathrm{kN} / \mathrm{m}$, after which the actual form-finding and beam relaxation is performed.

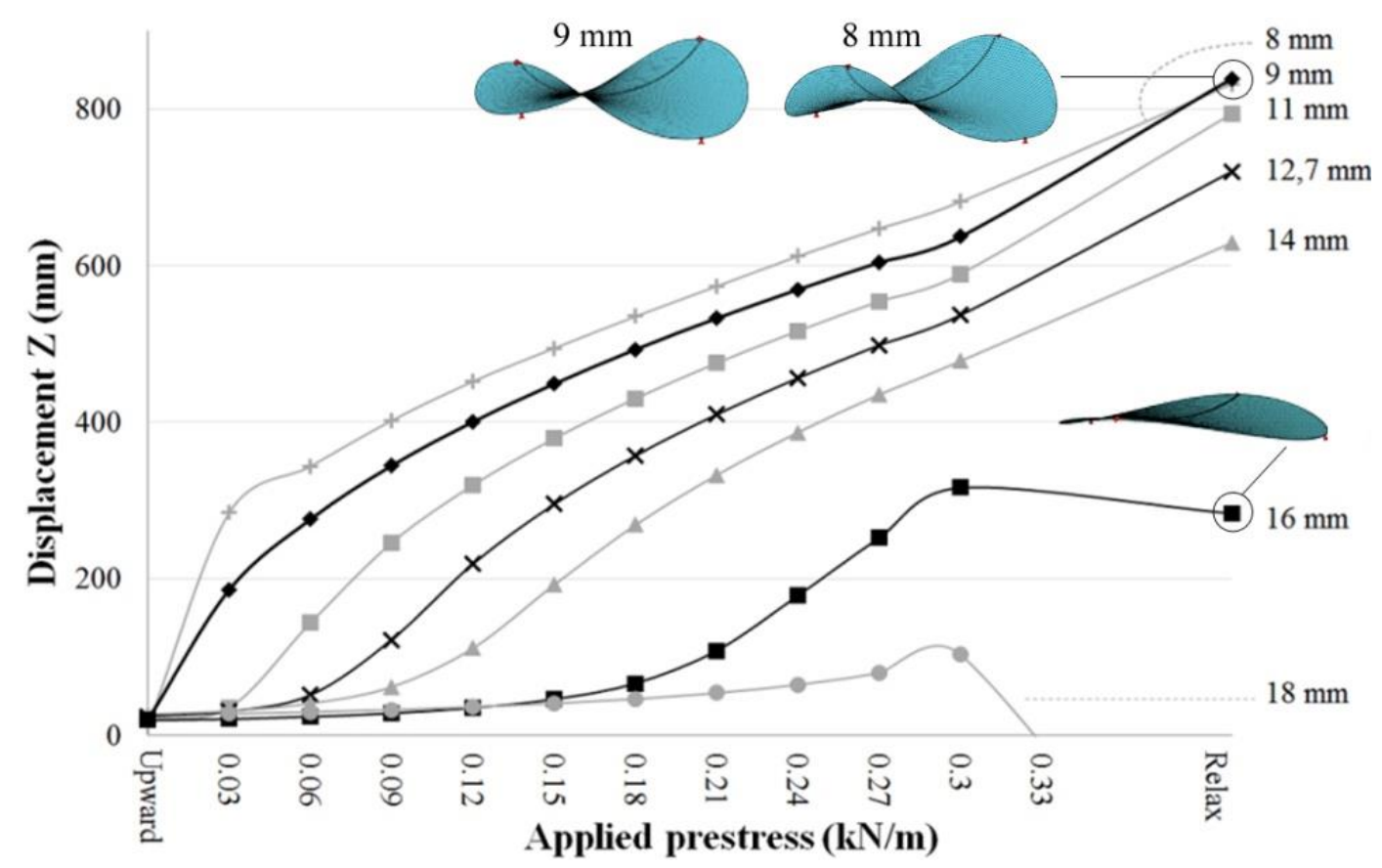

Figure 7. Position (Z) of the top points under a certain prestress for different beam sections 
The graph indicates that for the applied prestress of $0.3 \mathrm{kN} / \mathrm{m}$ and an increasing section, the out-of-plane displacement decreases until the prestress is insufficient to keep the pringle shape in position. At this point, the relaxation of the bending-active element no longer results in a further elevation of the top points, but in a drop down under the structure's self-weight (Figure 7: $16 \mathrm{~mm}$ and $18 \mathrm{~mm}$ ). When the beam section is chosen too small it 'fails' under the applied prestress, which results in a decrease in height of the top points (Figure 7: $8 \mathrm{~mm}$ ). The form-finding geometry of the structure with a boundary ring of $8 \mathrm{~mm}$ shows a different deformation, which is clearly visible when comparing the geometry to the one with a boundary ring of $9 \mathrm{~mm}$.

As mentioned before, the more the boundary ring deflects during the relaxation of the beam after the form-finding, the higher the difference is between the actual membrane forces and the intentional applied prestress value. Figure 8 shows the significant difference between four of the considered beam sections: for a section of $9 \mathrm{~mm}$ the forces in the centre drop from $0.3 \mathrm{kN} / \mathrm{m}$ to $\pm 0.1 \mathrm{kN} / \mathrm{m}$, whereas for the $18 \mathrm{~mm}$ section membrane forces of $\pm 0.28 \mathrm{kN} / \mathrm{m}$ are reached.

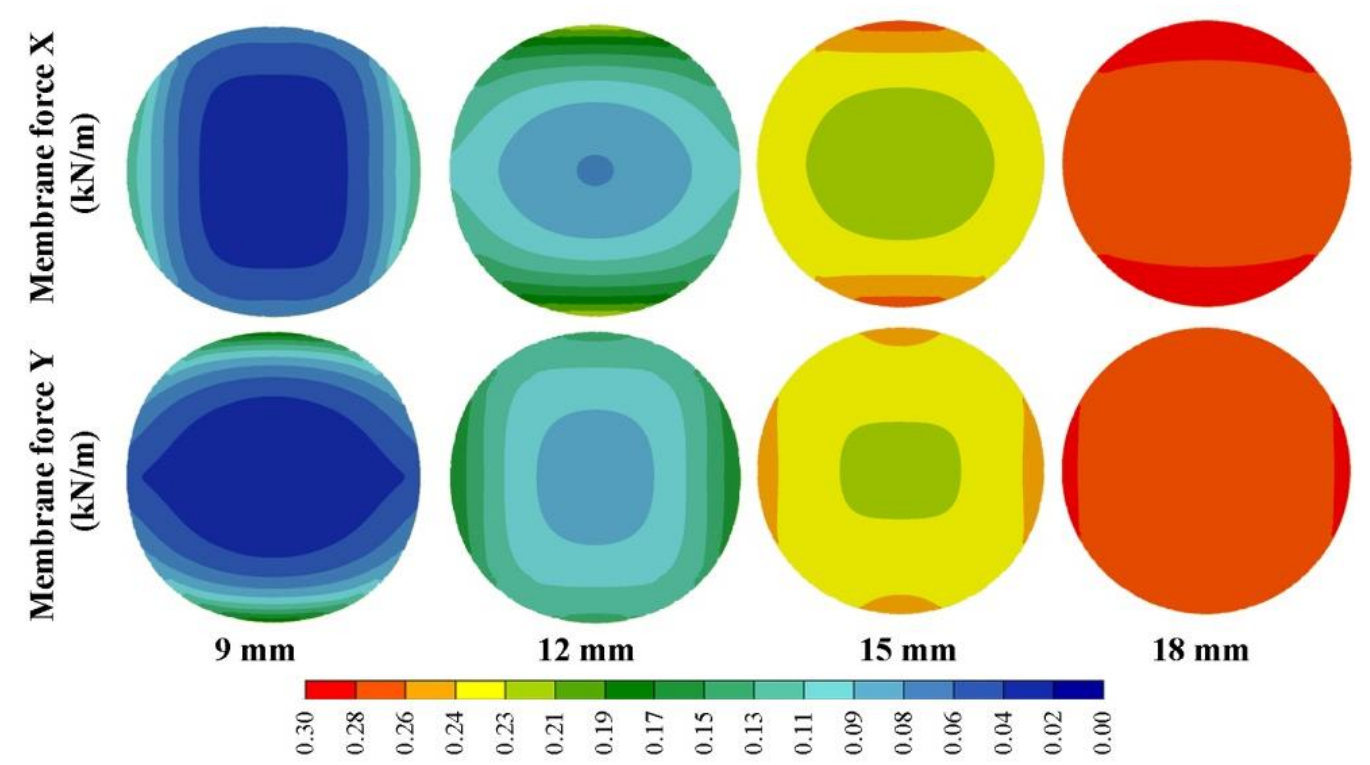

Figure 8. Membrane forces in $\mathrm{x}$ - and $\mathrm{y}$-direction $(\mathrm{kN} / \mathrm{m})$ at an intentional prestress of $0.3 \mathrm{kN} / \mathrm{m}$ for different beam sections

Nevertheless, considering that the diameter of the ring remains $3.18 \mathrm{~m}$, the residual stresses in the ring increase linearly with increasing beam section. From the three-point bending test a maximal stress of $600 \mathrm{MPa}$ is measured. Considering the material's safety factor ( $=1.3$ for machined profiles [9]), this results in a maximal design strength of $460 \mathrm{MPa}$ which might not be exceeded under any condition (even under e.g. wind loading, which is not yet considered in this stage).

It is thus very important to find a good balance between the chosen beam section and the applied prestress, as those parameters have great influence on each other. The selection of an appropriate prestress is further discussed in section 5.3.

Another design consideration is the ease of the kinetic deployment, which takes into account that the forces in the contraction cable increase to obtain the same rate of kinetic deployment, when choosing a greater beam section. 


\subsection{Internal elements}

Without an internal beam element, the centre point of the membrane is positioned at about half height of the top points after form-finding. Contracting the cable element between the top points generates a decrease in membrane length from one top point to the other top point, resulting in a reduction in strain (and consequently forces/stresses) in the membrane's y-direction (Figure 9, where a side view (yz) is given). Both due to the interaction between warp and weft direction and the geometric parameters, the forces in the membrane in $\mathrm{x}$-direction also decrease during the cable contraction.

To obtain a structurally stable system in all phases of the deployment, internal bending-active elements could be integrated in the pringle shape. For this first analysis, one internal beam element is added centrally in the initially flat circular structure, running from one top point to the other. The length of the internal element equals the diameter of the ring $(3.18 \mathrm{~m})$.
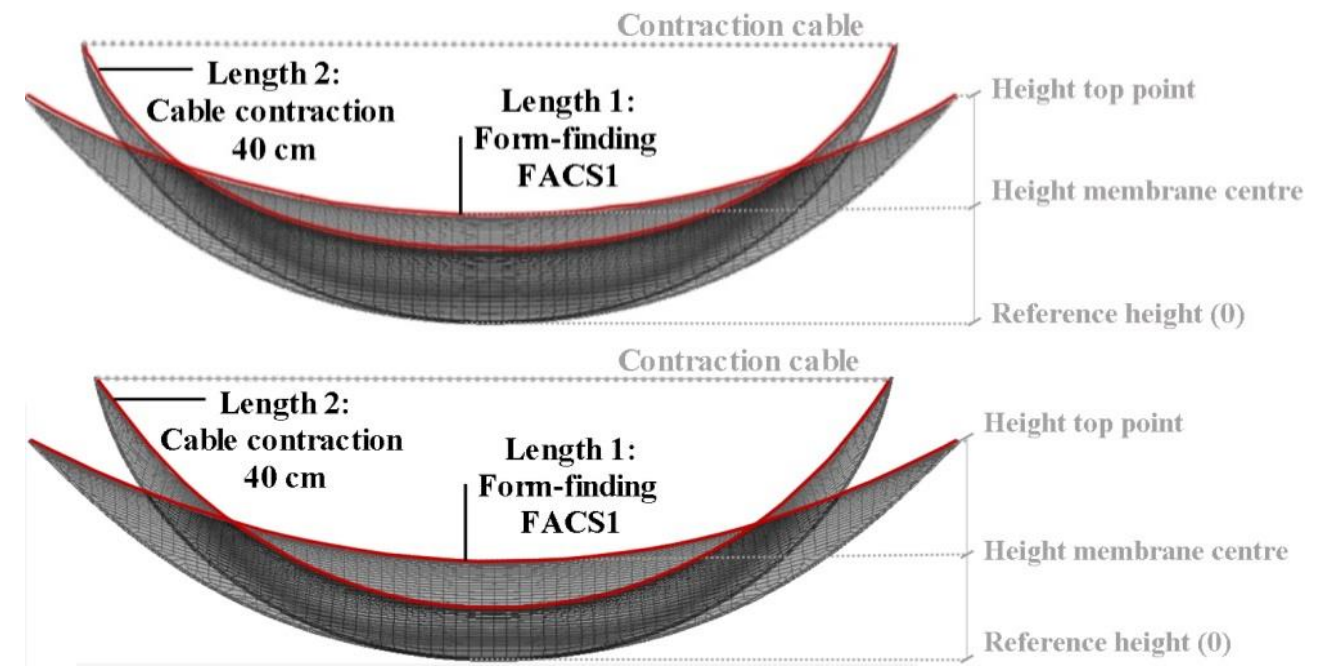

Figure 9. The membrane length between the top points reduces when shortening the contraction cable, causing a decrease in membrane forces - yz view: (top) situation without internal beam element; (bottom) with internal beam element

The addition of this internal element should avoid the quick loss of tension in the membrane, especially in the y-direction. As the pretension is applied and the top points move up- and inwards, the rectangular beam element will bend perpendicular to the xy-plane, pushing the membrane down. After the final form-finding, the centre of the membrane lies no longer at half height of the top points, but at $44 \%$ of the height of the top points. When the kinematic deployment is initiated, the internal element pushes the membrane down to keep the membrane stressed. In reality, the membrane will often slide along the internal beam element (the beam is placed in a pocket), causing a slightly different behaviour than the numerical model where the membrane is fully fixed to the internal beam element.

Figure 10 compares the forces in the membrane in both $\mathrm{x}$ - and $\mathrm{y}$-direction for the three cases: the pringle without an internal element, with an internal element that is fully fixed and with an internal element that allows sliding of the membrane.

In the form-finding state already slight improvements in membrane forces can be noticed with the internal element, especially in y-direction. When looking at the second state, where the contraction cable (initially with a length of $\pm 3 \mathrm{~m}$ ) is shortened with $\pm 20 \mathrm{~cm}$, and at the third state, where the cable is shortened with $\pm 40 \mathrm{~cm}$, it is proven that the loss of stresses in the y-direction is reduced. Unfortunately, the stresses in $\mathrm{x}$-direction consequently drop slightly when adding a beam element. 
Final form-finding Cable length

$($ cable $= \pm 3 \mathrm{~m}) \quad=2.79 \mathrm{~m}$
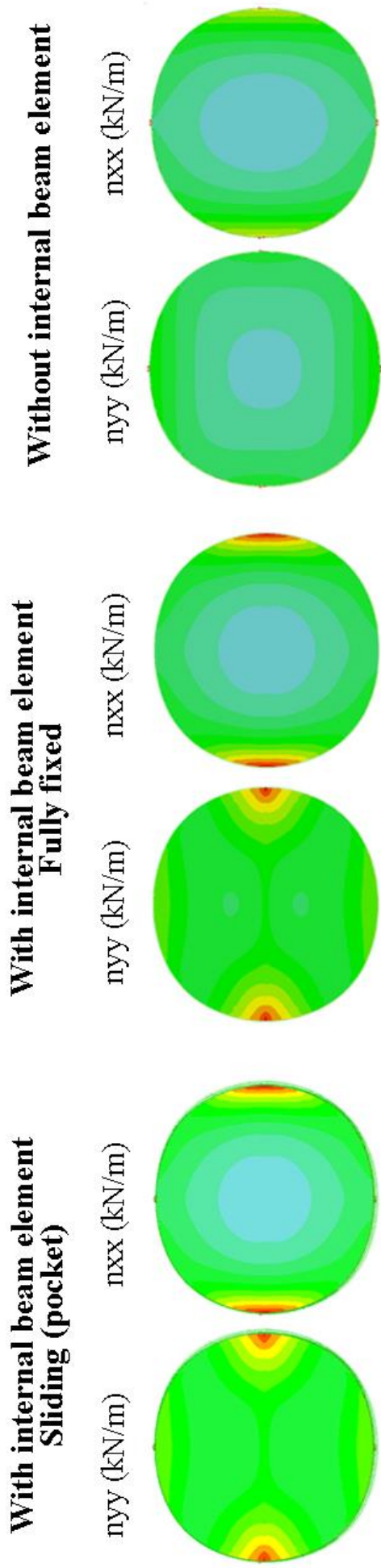

융 क्ष

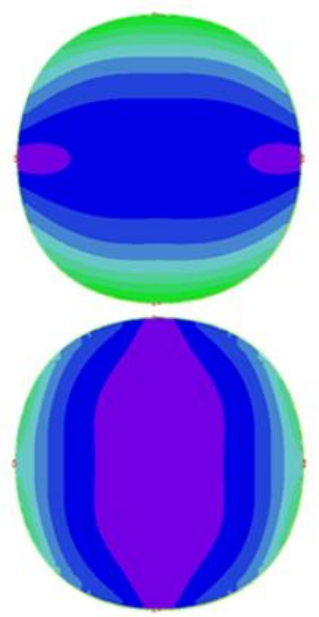

Cable length

$=2.61 \mathrm{~m}$
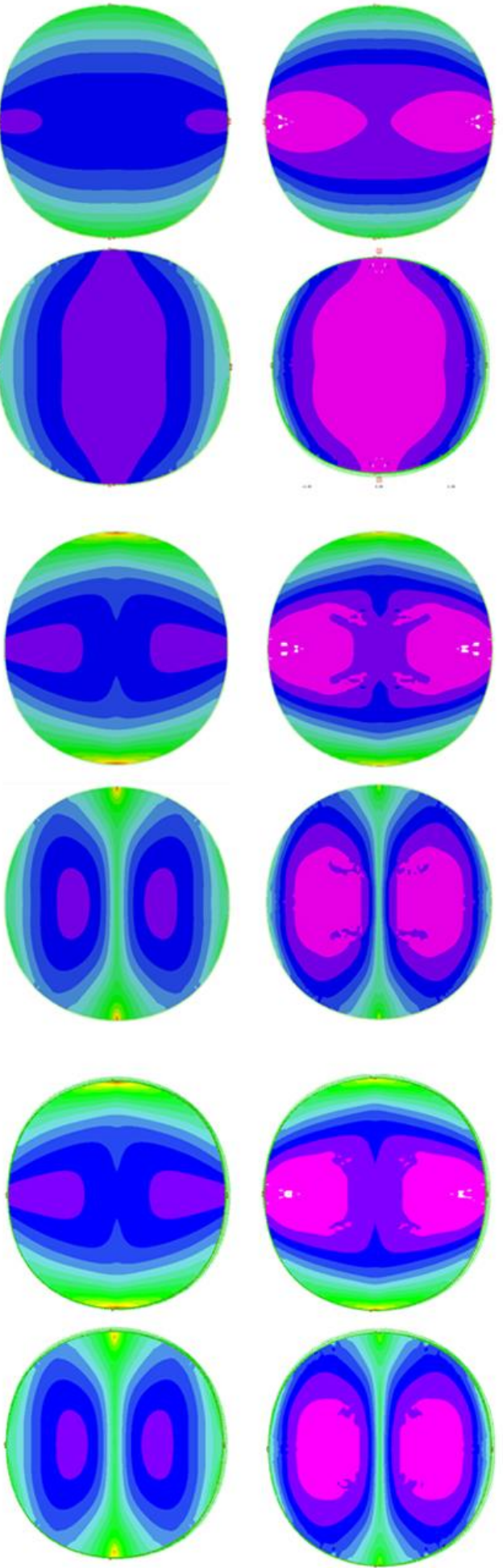

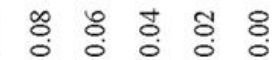

Figure 10. Membrane forces in $\mathrm{x}$ - and $\mathrm{y}$-direction $(\mathrm{kN} / \mathrm{m})$ for the pringle without internal beam element (row 1 and 2), with a fully fixed internal element (row 3 and 4) and a sliding internal element (row 5 and 6) 
As a second attempt, the intention is to increase the internal beam's length, so that after form-finding, the beam's middle point lies close to or under the reference height, i.e. the height of the lower points or 'zero level' (as indicated in Figure 9). In this case, shortening the contraction cable will push the internal beam element below the zero level, which results in positive straining of the membrane in $\mathrm{x}$-direction instead of losing prestress. Nevertheless, starting from a beam length that is longer than the ring's diameter would hinder the experimental investigation, as this wouldn't allow performing the DIC and other measurements starting from the flat untensioned reference state [12].

The differences in membrane forces for the fully fixed and the sliding beam are minimal, but for the 'experimental numerical model' (see [12]) the adoption of this sliding principle is required to match the real behaviour.

\section{3. $\quad$ Prestress in the membrane}

Similar to the variation of the ring's section with a constant membrane pretension, the variation of the membrane pretension using the same ring section results in different form-found geometries. The higher the pretension, the higher the top points are positioned after the form-finding (Figure 11). Analogous, the lower the prestress (and thus the smaller the ring deformations), the more homogeneously the forces in the membrane are distributed.

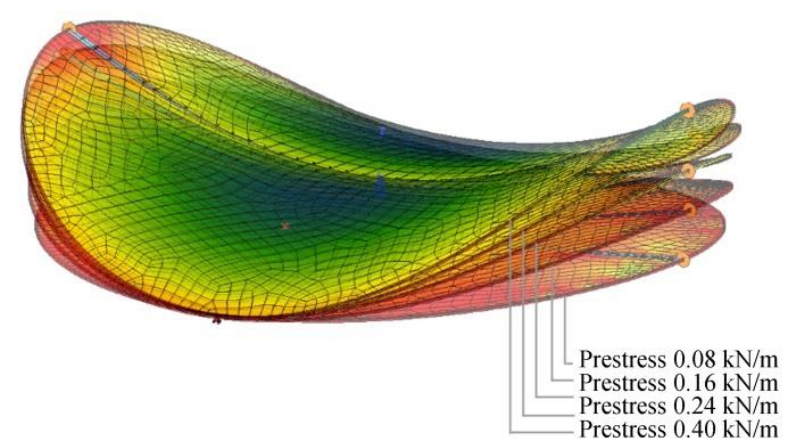

Figure 11. Deformation of the textile hybrid with an internal bending-active element with increasing initial prestress of the membrane

This can also be seen in the graphs in Figure 13, presenting the membrane forces in warp (y) and weft (x) direction along two lines. The horizontal line runs from one lower point to the other lower point. The vertical line runs in the middle of one half of the membrane (divided by the internal beam element), as the forces decrease the most in this area.

Also the behaviour during the kinematic deployment is highly influenced. Figure 12 shows the membrane stresses/forces for the situation where the contraction cable has a length of $2.61 \mathrm{~m}$ (LC32= load case 32). With a lower initial prestress the stress distribution in the membrane is more desirable, i.e. uniformly distributed and with a minimal reduction of the stresses, than with higher prestress values.

This can be related to the position of the central point of the internal beam element, which lies lower for a lower pretension, which is advantageous for the preservation of stresses in the $\mathrm{x}$-direction when shortening the contraction cable, as discussed in 5.2.

Nevertheless, it should be verified whether this state with a lower initial prestress is structurally stable enough under external loading, bearing in mind that the overall stiffness of membrane structures increases with increasing prestress and increasing curvature. 


\section{Von Mises Stresses membrane}

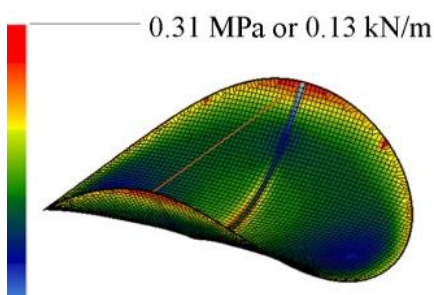

0.00

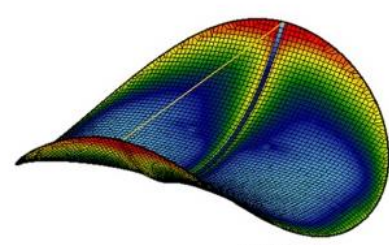

0.00
$0.73 \mathrm{MPa}$ or $0.31 \mathrm{kN} / \mathrm{m}$

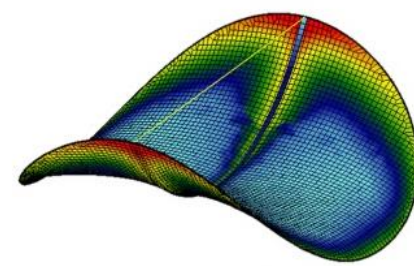

0.00

Figure 12. Membrane stresses (MPa)/forces $(\mathrm{kN} / \mathrm{m})$ when the contraction cable has a length of $\pm 2.61 \mathrm{~m}$; with an initial prestress of $0.2 \mathrm{kN} / \mathrm{m}$ (left), $0.3 \mathrm{kN} / \mathrm{m}$ (middle) and $0.4 \mathrm{kN} / \mathrm{m}$ (right)

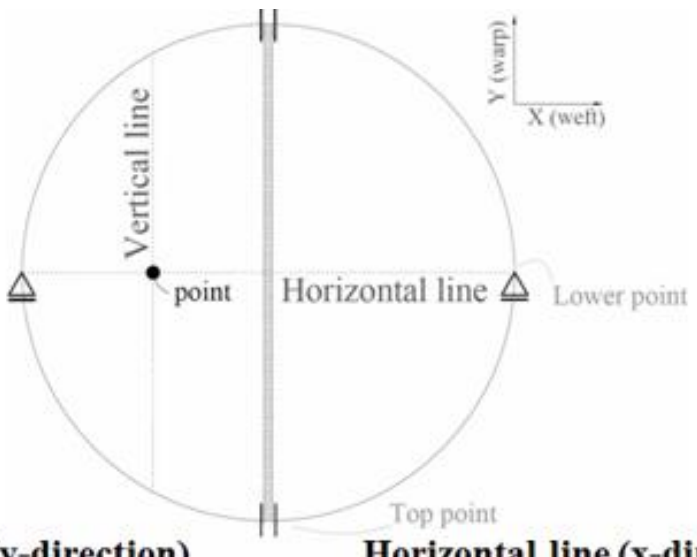

\section{Vertical line (y-direction)}

\section{Horizontal line (x-direction)}
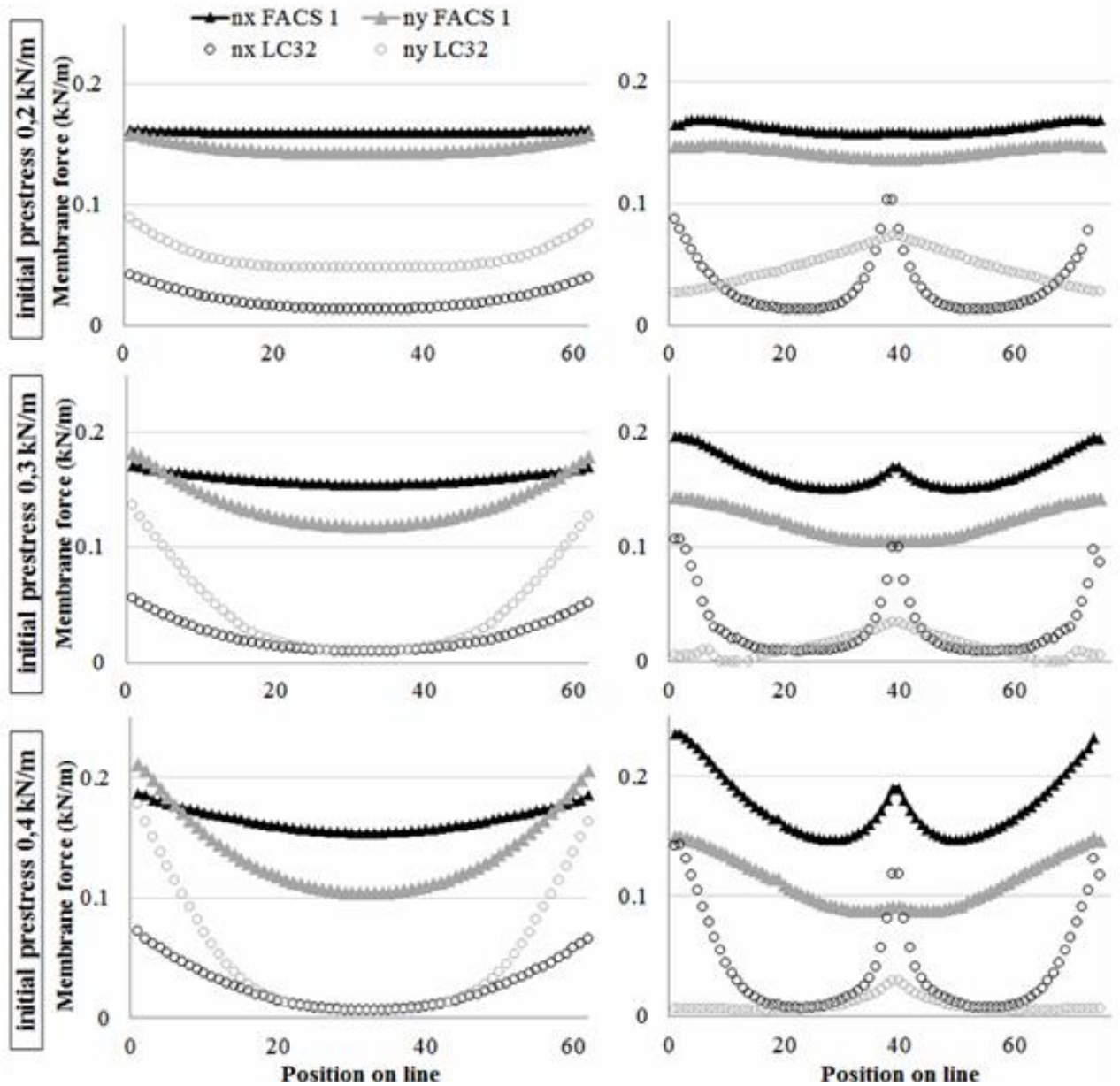

Figure 13. Membrane forces $(\mathrm{kN} / \mathrm{m})$ along two lines in $\mathrm{x}$ - and $\mathrm{y}$-direction, for the form finding state and for LC32 where the contraction cable has a length of $\pm 2.61 \mathrm{~m}$ (i.e. shortened with $\pm 40 \mathrm{~cm}$ ) 
As an example, the self-weight of the connection pieces in the experimental set-up are added to the form-found structure [12]. Note that the two lower points are fixed when the loading is applied. For a prestress of $0.3 \mathrm{kN} / \mathrm{m}$ a maximum vertical displacement of $16.7 \mathrm{~mm}$ is obtained under the applied loading, whereas for a prestress of $0.2 \mathrm{kN} / \mathrm{m}$ a maximum displacement of $33.2 \mathrm{~mm}$ is measured. This confirms that lowering the membrane prestress results in a decrease in overall structural stiffness.

Furthermore, an asymmetric prestress ratio is considered. Following the principles of the position of the internal beam element, one should choose the situation where the centre of the internal beam element is pushed down the most during form-finding.

Lowering the prestress in y-direction results in an increase in distance between the top points and consequently in an elevation of the centre point. Lowering the prestress in x-direction, on the contrary, causes an increase in distance between the lower points and a downward movement of the centre point, which is more advantageous for the further deployment of the structure (looking at the distribution of the membrane forces).

Table 3 points out some geometric parameters for three different prestress states: (i) the reference state of $0.3 \mathrm{kN} / \mathrm{m}$ in both warp and weft direction, (ii) a prestress of $0.2 \mathrm{kN} / \mathrm{m}$ in $\mathrm{x}-$ and $0.3 \mathrm{kN} / \mathrm{m}$ in $\mathrm{y}$ direction and (iii) a prestress of $0.3 \mathrm{kN} / \mathrm{m}$ in $\mathrm{x}$ - and $0.2 \mathrm{kN} / \mathrm{m}$ in y-direction.

Table 3. Geometric parameters for the different prestress ratios after form-finding, with warp- and weft-direction aligned with respectively $\mathrm{y}$ - and $\mathrm{x}$-direction

$\begin{array}{lcccc}\text { x \& y prestress } & \begin{array}{c}\text { Distance lower } \\ \text { points x (mm) }\end{array} & \begin{array}{c}\text { Distance top } \\ \text { points y }(\mathbf{m m})\end{array} & \begin{array}{c}\text { Height top points } \\ \mathbf{z}(\mathbf{m m})\end{array} & \begin{array}{c}\text { Height centre } \\ \text { point }(\mathbf{m m})\end{array} \\ \mathbf{0 . 3} \& \mathbf{0 . 3} \mathbf{~ k N} / \mathbf{m} & 2914 & 3055 & 706 & 312(=44 \%) \\ \mathbf{0 . 2} \& \mathbf{0 . 3} \mathbf{~ k N} / \mathbf{m} & 3100 & 3064 & 566 & 175(=31 \%) \\ \mathbf{0 . 3} \& \mathbf{0 . 2} \mathbf{~ k N} / \mathbf{m} & 2890 & 3093 & 654 & 321(=49 \%)\end{array}$

The membrane forces are plotted in function of the length of the contraction cable to study the decrease during the contraction (see Figure 14). The membrane forces are extracted in the central point of one half of the membrane (divided by the internal beam element), i.e. the intersection of the vertical and horizontal line indicated in Figure 13. The graphs indicate that the loss of membrane stresses is reduced when the prestress in $\mathrm{x}$-direction is lower than the prestress in $\mathrm{y}$-direction.
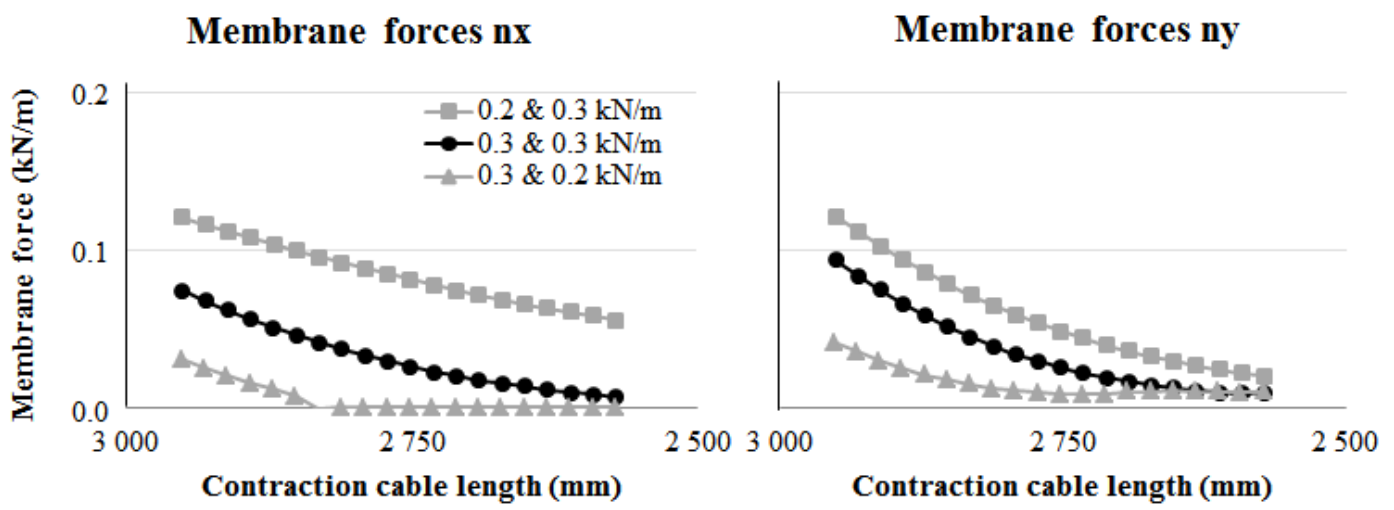

Figure 14. Membrane forces at the intersection of the vertical and horizontal line indicated in Figure 13 


\subsection{Orientation of the fibres}

The influence of the fibre orientation on the structural behaviour is verified by comparing three cases: the reference case aligns the warp direction with the internal beam element (i), the second case orients the weft direction along the internal element (ii) and in the third case the warp and weft directions are rotated $45^{\circ}$ (iii) (see Figure 15).
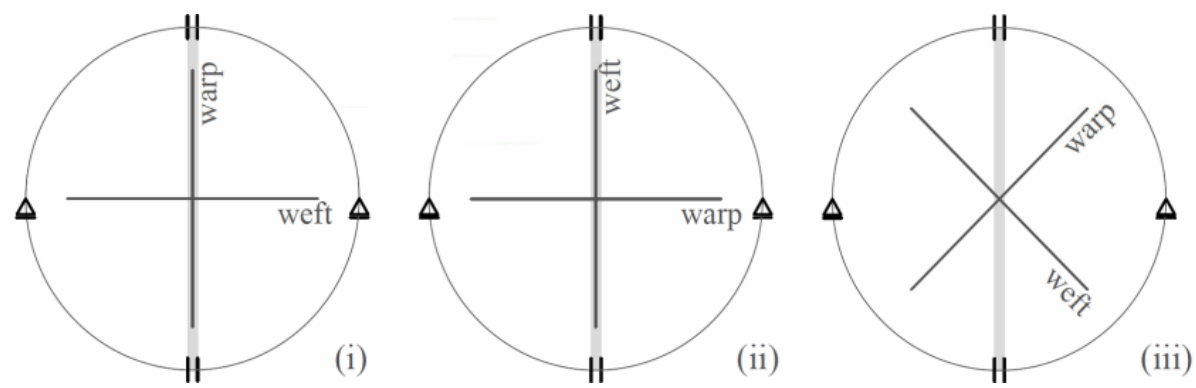

Figure 15. Indication of the studied fibre orientations

Figure 16 presents the membrane forces in $\mathrm{x}$ - and $\mathrm{y}$-direction, starting from an initial prestress of $0.3 \mathrm{kN} / \mathrm{m}$ in both warp and weft direction.

The $90^{\circ}$ rotated situation (Figure 16 (ii)) results in a more advantageous stress distribution during the kinematic deployment. As the warp direction will strain less to obtain the same prestress value and the weft direction deforms more, the distance between the lower points increases in this case and the distance between the top points decreases. This results in a ratio between the height of the top points and the height of the centre of the internal element that is lower (39.7\%) than in the reference case (44\%), which is beneficial.

Logically, rotating the membrane's warp and weft direction with $45^{\circ}$ results in an asymmetric stress pattern (see Figure 16 (iii)). The membrane forces are visualized along the warp- (x-) and weft- (y-) direction, as indicated in the figure. To compare the influence of this parameter, again the deflection under the self-weight of the connection pieces in the experimental model [12] is evaluated in the final form-finding state and in LC32. It needs to be noted that when loading the form-finding state, the two lower points are fully fixed and that in LC32, the contraction cable provides extra stiffness to the structure. For the reference case, the maximal deflection is $13.2 \mathrm{~mm} / / 14.2 \mathrm{~mm}$ (resp. form-finding // LC32). The case where the fibres are oriented at $45^{\circ}$ deflects $14.8 \mathrm{~mm} / / 16.1 \mathrm{~mm}$, which is thus less desirable. 

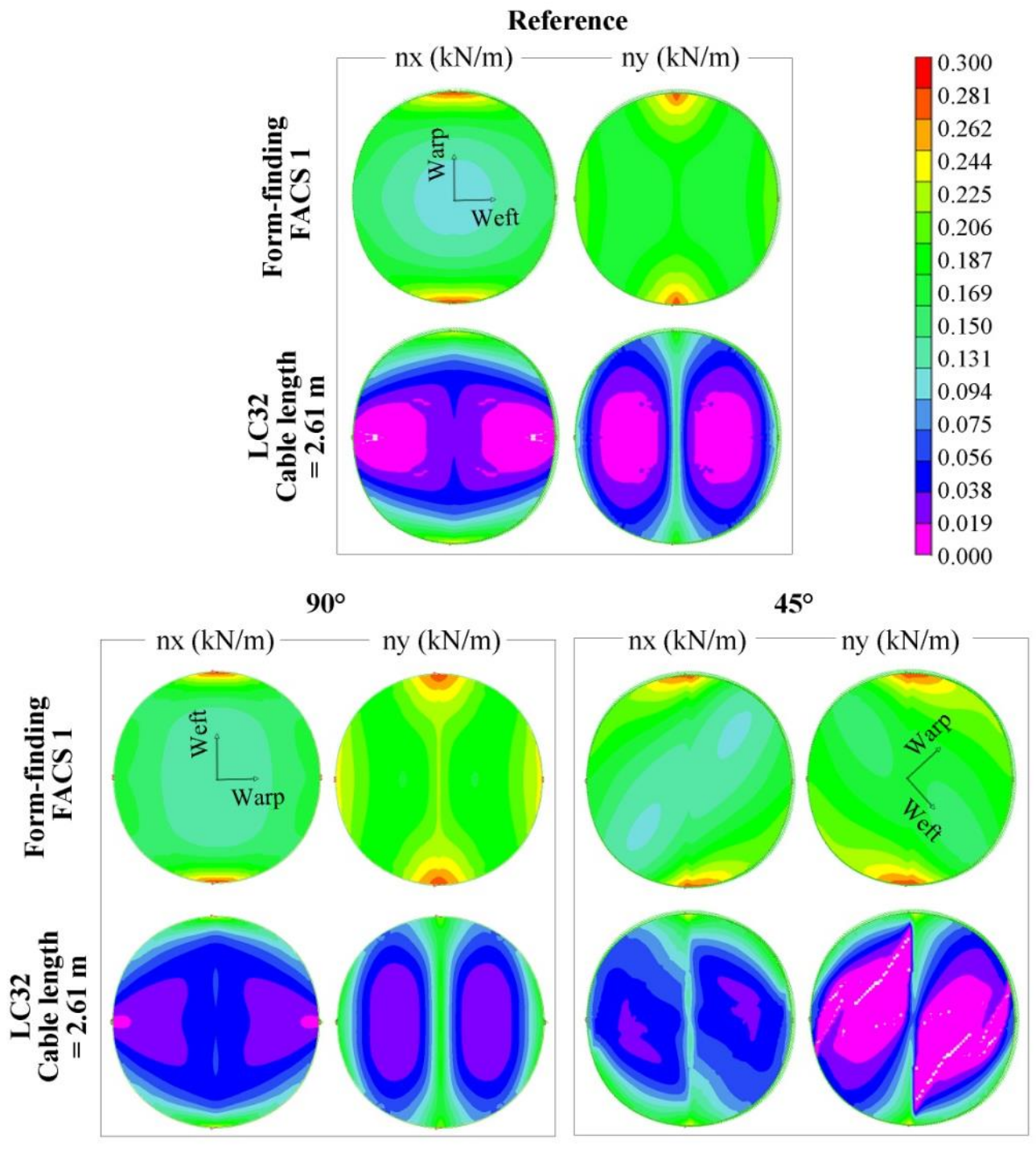

Figure 16. Membrane forces $(\mathrm{kN} / \mathrm{m})$ in $\mathrm{x}$ - and $\mathrm{y}$-direction for (i) the reference fibre orientation, i.e. with the warp direction aligned with the internal beam element, (ii) at $90^{\circ}$ and (iii) at $45^{\circ}$

\section{Conclusions}

As stated in the introduction, the objectives of the study are (1) to make the structural lightweight components work together, (2) be able to deploy and (3) keep the membrane tensioned (as uniformly as possible) to ensure a safe structural behaviour.

This parameter study illustrates the complexity of the structural behaviour due to the strong interaction between the ring, the beam, the cable and the membrane properties and the great influence on both the form-finding geometry and the behaviour during the kinematic deployment.

By comparing the forces in the membrane, the stresses in the beam elements, the overall geometry of the pringle shape and its deflection under a small external loading, the influence of a series of parameters is evaluated.

Due to the high deformation of the ring element under the applied membrane prestress, using a more flexible PU-coated polyester fabric improves the behaviour significantly, compared to using a relatively stiff PVC-coated polyester (please note that no realistic external loading is considered yet). The higher 
the membrane stiffness, the higher the loss in membrane stress for the same loss in strain, i.e. for the same ring deformation, which consequently leads to a less desirable stress distribution.

The bigger the ring's section (for the same applied prestress), the lower the ring deformation and thus the more homogeneous the stress distribution is. Nevertheless, the ring's initial bending stresses due to bending of an initially straight element will influence the designer's choice. Furthermore, the overall stiffness should be investigated, as it is known that for membrane structures both the prestress and the curvature of the membrane contribute to the overall structural stiffness.

The integration of an internal beam element improves the behaviour by pushing the membrane downwards, both for the form-finding state and during the deployment. The lower the middle point of the internal beam, the more advantageous the distribution of the membrane forces, which becomes important when analysing the influence of the other studied parameters.

Similar to varying the ring's section, changing the applied prestress in the membrane results in a variation of resulting geometries. The higher the prestress, the higher the curvature will be, but consequently the less homogeneous the membrane forces are distributed. Furthermore, the middle of the internal beam element lies lower for a lower prestress value, which proved more advantageous for the behaviour under deployment. Again, the important interaction between the different parameters is confirmed and thus also the importance of carefully considering different combinations. Also the choice of an asymmetric prestress is considered, where opting for a lower prestress in $\mathrm{x}$-direction than in $\mathrm{y}$ direction improves the structural behaviour.

Furthermore, the preferred orientation of the fibres can be selected based on the position of the middle point of the internal beam element. For this case study, aligning the stiffer fibre direction (i.e. the warp direction) with the line connecting the lower points (x-direction) leads to a more desirable structural response.

It can be concluded that the different studied parameters all have a great influence on each other and on the structural behaviour of the designed textile hybrid. This interaction complicates the design and thus the choice of the selected parameters. However, this integrated design model allows quantifying the influence of each parameter and helps the designer to make the appropriate structural design choices.

An experimental validation of the obtained transformable pringle-shaped textile hybrid is discussed in [12].

\section{Acknowledgements}

This PhD-research was conducted with the funding of the Research Foundation Flanders (FWO). Furthermore, the researchers would like to thank Sioen Industries for providing fabric material for the experimental investigation and the COST-Action TU1303 Novel Structural Skins for the possibility to join WG5 Materials and analysis.

\section{References}

[1] Puystiens, S., Van Craenenbroeck, M., De Laet, L., Van Hemelrijck, D., Van Paepegem, W., Mollaert, M., 2016. Integrated analysis of kinematic form active structures for architectural applications: Design of a representative case study. Engineering Structures, Volume 124, pp. 376-387. 
[2] Dieringer, F., Philipp, B., Wüchner, R. \& Bletzinger, K.-U., 2013. Numerical Methods for the Design and Analysis of Hybrid Structures. International Journal of Space Structures, 28(3\&4), pp. 149-160.

[3] Lienhard, J. \& Knippers, J., 2012. Permanent and convertible membrane structures with inricate bending active support systems. Seoul, Korea, Proceedings IASS Symposium 'From Spatial Structures to Space Structures'.

[4] Van Mele, T., De Laet, L., Veenendaal, D., Mollaert, M., Block, P., 2013. Shaping Tension Structures with Actively Bent Linear Elements. International Journal of Space Structures, 28(3), pp. 127-135.

[5] Lienhard, J., 2014. Bending-active structures: form-finding strategies using elastic deformation in static and kinetic systems and the structural potentials therein. Stuttgart, Germany: Institut für Tragkonstruktionen und Konstruktives Entwerfen (ITKE), PhD thesis.

[6] Sioen Industries. Coated technical textiles - Architectural Membranes. http://www.sioen.com/

[7] Galliot, C. \& Luchsinger, R. H., 2009. A simple model describing the non-linear bi-axial tensile behaviour of PVC-coated polyester fabrics for use in finite element analysis. Composite Structures, 90(4), pp. 438-447.

[8] Vink bvba. Plastic Solutions and more. http://www.vink.be/

[9] Fiberline Design Manual, Fiberline Composites, Denmark, 2003

[10] Dinh, T. et al., 2017. A hybrid micro-meso-scale unit cell model for homogenization of the nonlinear orthotropic material behavior of coated fabrics used in tensioned membrane structures. Composite Structures, Volume 162, pp. 271-279.

[11] Sofistik AG. Software for better Design. http://www.sofistik.com

[12] Puystiens, S., Van Craenenbroeck, M., De Laet, L., Van Hemelrijck, D., Van Paepegem, W., Mollaert, M., Implementation of bending-active elements in kinematic form-active structures - Part II: Experimental verification. Composite Structures (submitted) 